\title{
INDICADORES DE GESTIÓN EMPRESARIAL
}

Ing. Néstor Santos Jiménez

\section{RESUMEN}

Los inversionistas o accionistas de empresas requieren de indicadores que permitan apreciar la evolución, situación actual y perspectivas de la marcha operativa, económico financiera y patrimonial del negocio en que han comprometido su capital. Estos indicadores, que son índices o valores que relacionan las diversas actividades de la organización, permiten apreciar si la gestión de la empresa permite que ésta cumpla de manera satisfactoria con las exigencias de sus clientes, de modo que se garantice que el negocio sea sostenible en el tiempo, asegurando así al inversionista la recuperación de su capital con un retorno o rentabilidad adecuada, según el contexto en que se desenvuelve la empresa.

\section{ABSTRACT}

The investors or shareholders require of indicators that allow to appreciate the evolution, current situation, and perspectives of the financial and patrimonial operative, economic march of the business in that they have committed their capital. These indicators that are index or values that relate the diverse activities of the organization, allow to appreciate if the administration of the company satisfy the demands of their clients, so it is guaranteed that the business is sustainable in the time, assuring this way the investor the recovery of their capital with a return or appropriate profitability, according to the context in that it unwraps.

\section{INTRODUCCIÓN}

En toda actividad empresarial se requiere analizar el perfomance de la organización, sea ésta una empresa productiva o de servicios, de propiedad privada o con participación del Estado, especialmente en un contexto económico globalizado y que exige la competitividad de los negocios. Los cambios del entorno, que son permanentes, exigen que las decisiones sean oportunas y adecuadas. Para evaluar el éxito de un negocio se requiere de indicadores que permi$\tan$ medir el avance que se viene logrando.

En una empresa productiva, se mide el grado de aceptación de sus productos, la relevancia de las ventas en el sector en que se desarrolla el negocio y la evolución en el tiempo de estos indicado res. Por ello, términos como porcentaje de participación en el mercado, diversificación de ventas según estratos y crecimiento periódico de ventas, facilitan percibir el avance que viene logrando el negocio. Pero, también interesa conocer cómo vamos con nuestros clientes internos, los trabajadores y los accionistas; por lo que se utilizan índices como rotación del personal, capacitación del trabajador, uso de capacidad instalada de las instalaciones, rendimiento de las inversio nes, valor contable y valor de mercado de las acciones representativas del capital social de la empresa.

Estas mismas reflexiones son también aplicables en empresas de servicios, por ejemplo de mensajería, telecomunicaciones, educación, servicio eléctrico, por mencionar algunas. En el caso de servicios públicos, como el suministro de energía eléctrica, existen elementos adicionales normados por la legislación del sector, los cuales también se dan en determinados sectores productivos, pero en menor grado.

De otro lado, si bien el Estado, en el esquema del actual gobierno, tiende a desarrollar un rol promotor, propiciando que la actividad empresarial esté a cargo del sector privado, aún se tienen negocios en donde se da la consistencia entre empresas de propiedad del Estado y empresas privadas. A pesar de toda la actividad empresarial del Estado está centralizada en una sola entidad, el FONAFE, aún se presentan algunas discrepancias en la gestión de empresas en
"Para evaluar

el éxito de un negocio se requiere de indicadores que permitan medir el avance que se viene

logrando." 


\section{GESTIÓN EMPRESARIAL}

donde el Estado tiene el 100\% de participación accionaria destacándose aquí normas de control, que tienden a flexibilizarse, pero que regulan lo que la empresa "debe hacer" y lo que "no debe hacer"; también se tiene la eventual influencia política del gobierno. Por lo tanto, la interpretación de los indicadores de gestión debe considerar esta especial situación del entorno.

En un esquema básico de negocios, un inversio nista aporta un determinado capital y constituye una empresa. En algunos casos, dicho aporte es complementado con recursos financieros de terceros vía endeudamiento. La inversión inicial para concretar el negocio, de esta manera, será financiada con deuda de acreedores y aporte de capital del inversionista. El encargado de la gestión del negocio, a través de las instancias societarias que correspondan, permanentemente analiza los logros de la empresa. En las pequeñas empresas este análisis es implícito, al estilo de cada inversionista. En empresas medianas y grandes, el análisis de negocios da lugar a que estas tareas sean más objetivas y que en muchos casos cuentan con unidades orgánicas especializadas, como Planeamiento, Desarrollo o Control de Gestión.
En todo tipo de negocios es innegable la necesidad de realizar el control de la gestión, en términos de hacia dónde se debe conducir el negocio, qué acciones previas se deben realizar, cómo analizar los resultados y qué ajustes requiere la organización. Estas inquietudes son permanentes, toda vez que el cambio es algo innato en la actividad humana y por ende en todo negocio. Sólo varían los estilos de gestión, en función a los dueños, directores, gerentes y los trabajadores en general.

Para una evaluación sistemática de la empresa, es importante dominar el entorno externo e interno de cada organización. Del contexto externo, se requiere conocer la situación y perspectivas del país, en los aspectos políticos, económicos y sociales, así como del sistema financiero y mercado de capitales. Desde el punto de vista interno, es necesario dominar los aspectos operativos, económico financieros y societarios. Es un conocimiento interrelacionado con flujo de información en ambos sentidos: del entorno externo al medio interno y viceversa, según el esquema relacional que se muestra en el diagrama $\mathrm{A}$.

Diagrama A

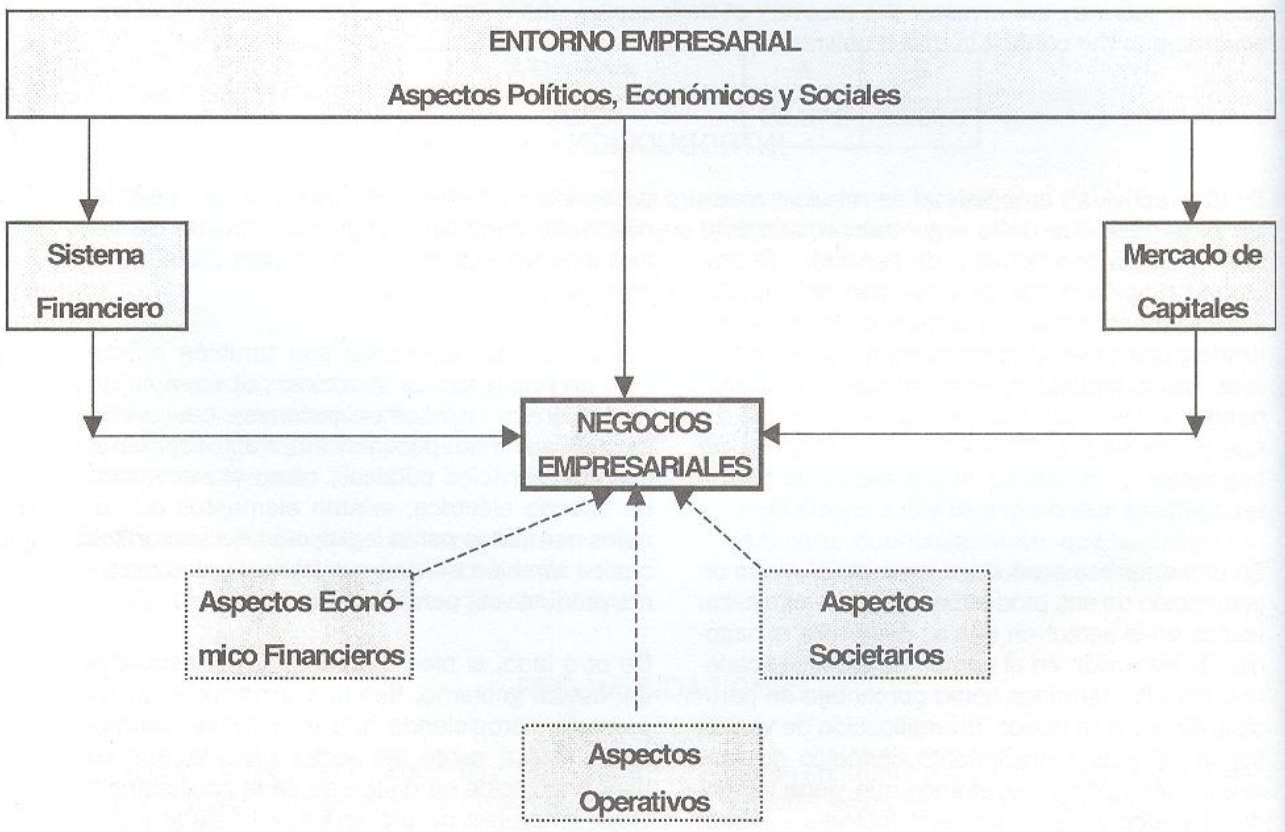

A continuación se muestran determinados indicadores de gestión, con evidencias aplicadas en diversas actividades empresariales. Se hace énfasis en los aspectos operativos, que son la base para explicar la situación económica, financiera y patrimonial de una empresa. 


\section{INDICADORES OPERATIVOS}

Los aspectos operativos del negocio están reflejados en la evolución de ventas, la producción y los inventarios de productos terminados, así como en la capacidad instalada, innovaciones técnicas y la administración. Esta situación se presenta fundamentalmente en empresas productoras manufactureras, en donde la producción obtenida durante un determinado período se almacena para su venta, según la tecnología disponible y la administración aplicada. Este esquema es igualmente válido para empresas que trabajan en función a pedidos $u$ órdenes de trabajo, que es el caso típico de talleres de manufactura. Es un esquema de operación que comprende tres actividades básicas: comprar, producir y vender.

Esta misma lógica se da en empresas de servicios. La particularidad del negocio definirá los indicadores a ser utilizados para decidir si la gestión que se viene aplicando es la más adecuada; también dependerá del interés en realizar cambios que permitan obtener mejores lo gros. Asimismo, además de analizar la evolución cronológica de estos indicadores, es deseable realizar comparaciones con los resultados de la competencia; para lo cual se requiere que la información sobre las empresas sea pública.
Entre los principales indicadores de carácter operativo, se puede mencionar a: participación en el mercado; estructura de ventas por segmentos de mercado $\mathrm{y} / \mathrm{o}$ por distribuidores $\mathrm{y} / \mathrm{o}$ por vendedores directos; porcentaje de ventas devueltas; número de reclamos de clientes; productividad de la materia prima, de la mano de obra, de la maquinaria y equipos, y de otros insumos; y uso de la capacidad instalada.

Como ilustración, para explicar los principales indicadores operativos de un negocio que presta servicios públicos, se tiene la información sobre el Sistema Privado de Pensiones, producto que en nuestro país tiene ya siete años y que se encuentra en su fase de consolidación. La información correspondiente al 30 de setiembre de 2000 , se muestra en el cuadro $\mathrm{N}^{\circ} 1$, se refiere al número de afiliados (clientes), valor de la cartera administrada (inversión de los afiliados), promedio de cartera por afiliado (inversión realizada por cada cliente) y productividad del mes (nuevos afiliados captados por cada promotor contratado). Esta información es publicada mensualmente por la Superintendencia de Banca y Seguros, la cual en el presente año ha absorbido a la Superintendencia del Fondo de Pensiones.
“En todo tipo de negocios es innegable la necesidad de realizar el control de la gestión, en términos de hacia dónde se debe conducir el negocio..."

Cuadro $\mathrm{N}^{\circ} 1$

\section{Sistema Privado de Pensiones} A 30 de Setientore de 2000

\begin{tabular}{|c|c|c|c|c|c|}
\hline \multirow[b]{2}{*}{ Concepto } & \multicolumn{4}{|c|}{ Administradora del Fondo de Pensiones (AFP) } & \multirow[b]{2}{*}{ SPP } \\
\hline & Horizonte & Integra & UniónVica & Profuturo & \\
\hline N Total de Afiliados & $\begin{array}{c}632535 \\
26 \%\end{array}$ & $\begin{array}{c}611148 \\
25 \%\end{array}$ & $\begin{array}{c}595311 \\
25 \%\end{array}$ & $\begin{array}{c}5 / 5172 \\
24 \%\end{array}$ & $\begin{array}{c}2414166 \\
100 \%\end{array}$ \\
\hline Valor de la Cartera Administrada (miles SI.) & $\begin{array}{c}2392600 \\
25 \%\end{array}$ & $\begin{array}{c}3020517 \\
32 \%\end{array}$ & $\begin{array}{c}2735493 \\
29 \%\end{array}$ & $\begin{array}{c}1409665 \\
15 \%\end{array}$ & $\begin{array}{c}95582 / 5 \\
100 \%\end{array}$ \\
\hline Promedio Cartera/ Afiliado (SI/Afiliadb) & 3783 & 4942 & 4595 & 2451 & 3959 \\
\hline Productividad Promedio del Mes & 31,73 & 21,29 & 14,9 & 9,24 & 17,79 \\
\hline
\end{tabular}




Página 62
INDUSTRIAL DATA

\section{Participación en el Mercado}

Este indicador mide el grado de penetración del negocio en el mercado que atiende y está representado por el cociente entre las ventas físicas y las ventas de todas las empresas del sector o actividad económica al cual pertenece el negocio. El cálculo se realiza a valores físicos.

En el caso del Sistema Privado de Pensiones, el mercado total está formado por los trabajadores dependientes que han optado por esta modalidad de "ahorro obligatorio" para cuando se jubilen, según condiciones fijadas por la 30 de setiembre de 2000 , se tiene un poco más de 2,4 millones de afiliados ( $9 \%$ de la población del país), cuyo aporte al sistema asciende a cerca de S/.9 558 millones, que equivale a US $\$ 2731$ millones y representa aproximadamente el $5 \%$ del Producto Bruto Interno. Con este aporte de los trabajadores, las AFP's realizan inversiones en entidades financieras y empresas, dentro de los alcances que la Legislación establece sobre el particular; por lo que la contribución de este fondo al desarrollo del país es innegable.

Se observa que, en términos del número de afiliados, prácticamente las cuatro Administradoras del Fondo de Pensiones (AFP's) tiene la misma participación, cada una con alrededor del $25 \%$ del mercado, según se observa en el diagrama $\mathrm{B}$.

Diagrama B

“... además de analizar la evolución cronológica de estos

indicadores, es deseable realizar comparaciones con los resultados de la competencia..."

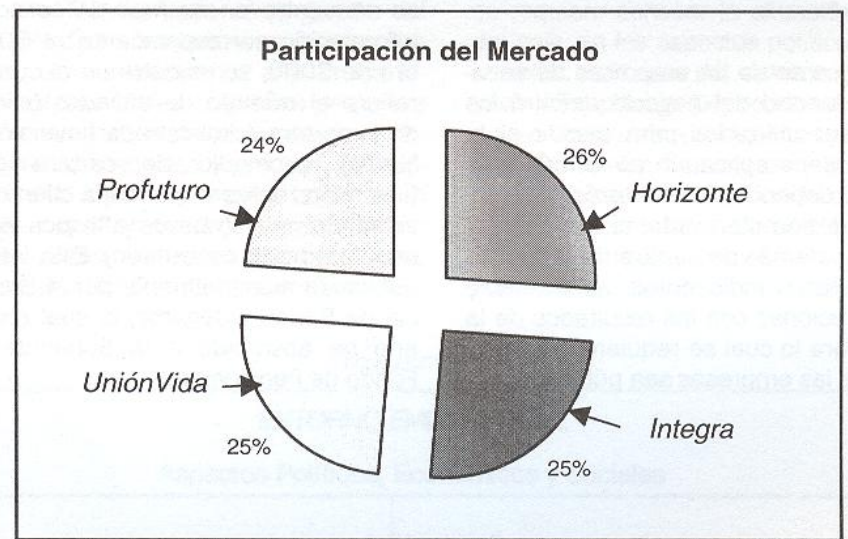

Sin embargo, esta participación a nivel del valor de la cartera administrada (aporte de los afiliados) varía desde el $15 \%$ (Profuturo) hasta $32 \%$ (Integra). Las otras AFP's tienen una participación de $25 \%$ (Horizonte) y $29 \%$ (UnionVida). Esta diferencia se explicaría por el valor promedio de la cartera por afiliado, la rentabilidad que se viene obteniendo y el grupo empresarial que respalda a cada AFP.

\section{Productividad del Personal}

La combinación óptima entre la infraestructura productiva de una empresa (inmuebles, maquinaria y equipos, e instalaciones) y el número de trabajadores, es aquella que produce los mejores resultados para el negocio, los que al final se van a traducir en ganancias y por ende en el valor de las acciones o títulos valores representativos del capital social de la empresa.

Dadas las diversas modalidades de contratación de personal que permite la Legislación y la forma en que se organiza una empresa, se debe tener especial cuidado en el cálculo de la pro- ductividad del personal y con mayor razón si se va a comparar el índice de las empresas que participan en el sector seleccionado y si una es empresa privada y la otra es empresa de propiedad del Estado. Esto no debe entenderse que una empresa estatal no es eficiente, especialmente en un contexto de libre competencia, sino que las diferencias que se observen requieren mayor análisis para su adecuada interpretación.

Para el ejemplo que estamos presentando, de Fondo Privado de Pensiones, la Productividad por Trabajador se mide en función al número de nuevos afiliados al sistema y el número de promotores utilizados. Es decir no se considera a todo el personal de la AFP y a la totalidad de afiliados, toda vez que estos valores no reflejan los logros del período, que es lo que se quiere evaluar. Para el mes de setiembre de 2000 , la productividad, medida por el número promedio de nuevos afiliados incorporados al Sistema Privado de Pensiones (sin considerar traspasos) y la cantidad de promotores utilizados, se presenta en el diagrama C. 


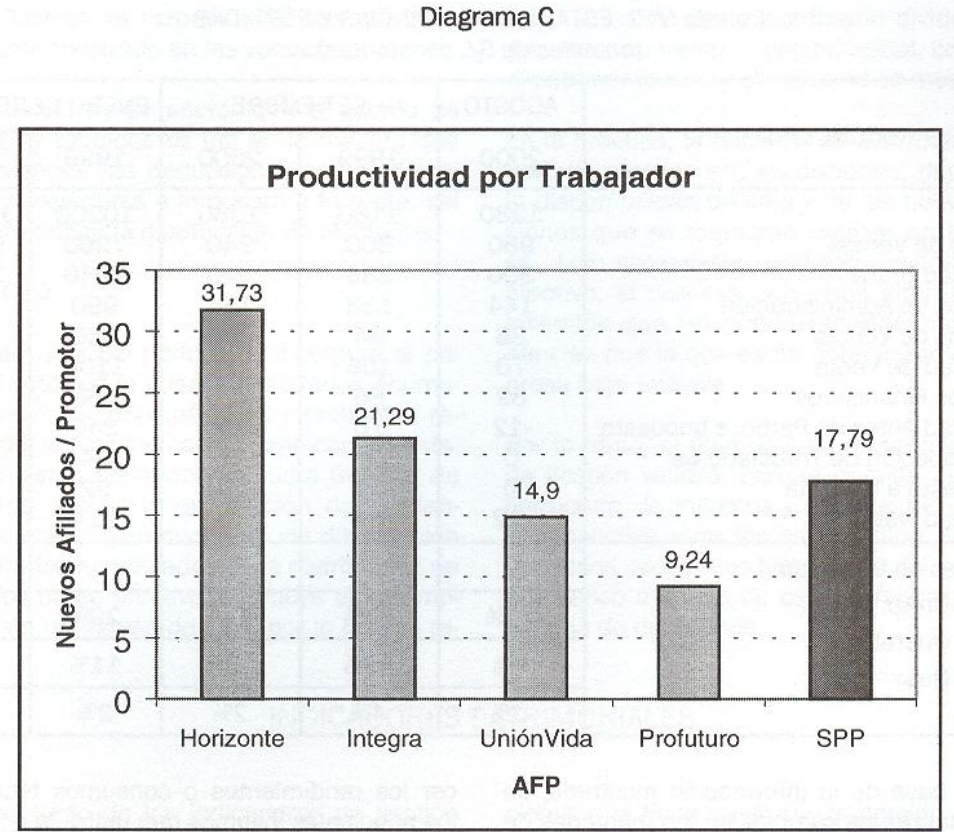

Se puede observar que la productividad del Sistema Privado de Pensiones (SPP) en setiembre de 2000 fue de 17,79 nuevos afiliados por ca- da promotor empleado. Horizonte e Integra superan el promedio y UniónVida y Profuturo registran índices menores.

\section{“Los resultados finales de un negocio, como consecuencia de los aspectos operativos del mismo, se traducen en los estados \\ financieros ..."}

Los resultados finales de un negocio, como consecuencia de los aspectos operativos del mismo, se traducen en los estados financieros, siendo los principales, el estado de ganancias y pérdidas, el flujo de caja y el balance general; los cuales, usualmente se elaboran en forma mensual, trimestral y anual.

El estado de ganancias y pérdidas y el flujo de caja, miden los resultados económicos y financieros del negocio, respectivamente. Los valores que se muestran corresponden al periodo corriente, al período inmediato anterior, los valores acumulados del ejercicio económico corriente y lo comparativo del mismo periodo del ejercicio anterior. En cambio, el balance general, por su naturaleza, es acumulativo y permite apreciar la situación patrimonial de la empresa a una fecha determinada. Las comparaciones, entre cuentas contables de diferentes periodos, deben realizarse a valores constantes de la última fecha.
Para analizar la situación económica, financiera y patrimonial de una empresa se utilizan los ratios o índices financieros, los cuales se agrupan en índices de liquidez, de solvencia, de gestión y de rentabilidad. Estos indicadores relacionan las principales cuentas de los estados financieros y para su interpretación, además, se utilizan los indicadores operativos mencionados anteriormente. En el presente artículo no se analizan todos los índices financieros, sino sólo los más usuales y que definitivamente, para su adecuada interpretación, requieren de indicadores operativos.

En el cuadro $\mathrm{N}^{\circ} 2$, con fines de ilustración, se muestra una estructura global del estado de ganancias y pérdidas de una empresa genérica, del mes de setiembre de 2000. Para su análisis, se incluye los valores del mes anterior (agosto de 2000), del mismo mes del año pasado (setiembre 1999) y el acumulado al mes de setiembre de 2000 y enero - setiembre de 1999. Estas cifras están expresadas a valores constantes del 30 de setiembre de 2000 . 
Cuadro $N^{\circ}$ 2. ESTADO DE GANANCIAS Y PÉRDIDAS (en miles de S/. constantes)

\begin{tabular}{|l|c|c|c|c|c|}
\hline \multirow{2}{*}{\multicolumn{1}{|c|}{ CONCEPTO }} & AGOSTO & \multicolumn{2}{c|}{ SETIEMBRE } & \multicolumn{2}{c|}{ ENERO-SETIEMBRE } \\
\cline { 2 - 6 } & 2000 & 1999 & 2000 & 1999 & 2000 \\
\hline Ventas & 1280 & 1186 & 1360 & 10200 & 12800 \\
(-) Costo de Ventas & 980 & 800 & 940 & 7260 & 9240 \\
Utilidad Bruta & 300 & 386 & 420 & 2940 & 3560 \\
(-) Gastos de Administración & 144 & 136 & 154 & 980 & 1280 \\
(-) Gastos de Ventas & 86 & 94 & 94 & 860 & 760 \\
$\quad$ Utilidad de Venta & 70 & 156 & 172 & 1100 & 1520 \\
(-) Gastos Financieros & 82 & 86 & 120 & 760 & 1020 \\
$\quad$ Utilidad Antes de Partic. e Impuesto & -12 & 70 & 52 & 340 & 500 \\
(-) Participación de Trabajadores & 0 & 7 & 5 & 34 & 50 \\
(-) Impuesto a la Renta & 0 & 19 & 14 & 92 & 135 \\
$\quad$ Utilidad Neta & -12 & 44 & 33 & 214 & 315 \\
\hline Márgenes de Ganancia: & & & & & \\
\hline Margen Bruto & $23 \%$ & $33 \%$ & $31 \%$ & $29 \%$ & $28 \%$ \\
\hline Margen Operativo & $5 \%$ & $13 \%$ & $13 \%$ & $11 \%$ & $12 \%$ \\
\hline Margen Neto & $-1 \%$ & $4 \%$ & $2 \%$ & $2 \%$ & $2 \%$ \\
\hline
\end{tabular}

Sobre la base de la información mostrada, se puede analizar los cambios en los márgenes de ganancia y en el crecimiento (variación porcentual por período de las principales cuentas); para cuya interpretación será conveniente contar con el detalle de las ventas físicas y de la estructura de costos y gastos.

\section{Márgenes de Ganancia}

Estos indicadores consideran que las ventas equivalen al $100 \%$ y qué porción de ella es explicada por las utilidades contables obtenidas, llámese utilidad bruta, utilidad operativa y utilidad neta; y así tenemos, margen bruto, margen operativo y margen neto, respectivamente. Estos son indicadores relativos, los que deben interpretarse conjuntamente con los valores absolutos correspondientes.

En el ejemplo, se aprecia que el margen neto del mes de setiembre de 2000 es $2 \%$ (de las ventas), que mejora con relación al mes pasado $(-1 \%)$, pero que es menor que el obtenido en setiembre del año 1999. En términos acumulados, de enero a setiembre, en el año 2000 , el margen es similar al obtenido en el mismo período del año 1999. Estos valores están explicados por las variaciones registradas en los márgenes de ganancia operativo y bruto, además de las ventas físicas y estructura analítica de costos y gastos de la empresa, y de ser el caso. La interpretación mejora si se tiene el desagregado por líneas y/o tipos de productos, áreas y/ o plantas de producción, zonas del mercado y/o agentes vendedores; incluso, se requiere cono- cer los rendimientos o consumos técnicos de los principales insumos que utiliza la empresa.

\section{Índices de Crecimiento}

Otro aspecto que facilita percibir la evolución del negocio, es la variación porcentual de las cuentas contables con respecto al tiempo. Según el cuadro $\mathrm{N}^{\circ} 2$, tenemos que las ventas de setiembre de 2000 se han incrementado en $6 \%$ con relación al mes de agosto, en cambio los costos y gastos totales han aumentado en sólo $1 \%$, lo cual explica la mejora en las utilidades y por ende en el margen neto.

Asimismo, en el referido cuadro se aprecia que las ventas acumuladas de enero a setiembre de 2000 se han elevado en $25,5 \%$ con respecto al mismo período de 1999; para dichos plazos, los costos y gastos también aumentan en $24,7 \%$, por lo que la utilidad neta del año 2000 , en términos absolutos aumenta de manera importante, es $47 \%$ mayor que el registrado en 1999. Pero, este margen, como proporción de las ventas de cada ejercicio es el mismo ( $2 \%$ de las ventas).

Un detalle interesante es que la venta promedio mensual registrada en el año 2000 es mayor en $25 \%$ que la obtenida en el ejercicio 1999. Este aumento no guarda una relación clara con las ventas de los meses de agosto y setiembre. Es posible que la variabilidad observada sea aleatoria, en todo caso, un análisis del sistema operativo (productivo, comercial y administrativo) de la empresa y las características del entorno 
podrían ilustrar las razones objetivas del comportamiento mostrado en las ventas.

De otra parte, es de anotar que el estado de ganancias y pérdidas es útil en la medida que permite estimar las deducciones por participación de trabajadores e impuesto a la renta, así como para definir la distribución de utilidades.

\section{Flujo de Caja}

La utilidad neta del período se incorpora al patrimonio neto, en la cuenta Resultados Acumulados, luego de cubrir pérdidas y realizar la reserva legal, si fuese el caso. Luego con los estados financieros auditados, la Junta General de Accionistas decide la distribución de dividendos, en efectivo o en acciones, vía disminución de Resultados Acumulados. Si la distribución de dividendos es en efectivo, diminuye el patrimonio y existe un egreso de caja, por lo que se re-

duce el activo. Si se distribuyen dividendos en acciones, aumenta el capital social, con lo cual el patrimonio neto y el activo no se modifican.

En la práctica, la decisión de distribuir dividendos, en efectivo y/o en acciones, depende de la disponibilidad de caja y de las nuevas inversiones que se requieran realizar en la empresa. Los accionistas prefieren dividendos en efectivo, si cuentan con otras alternativas de inversión que les podría reportar mayores ganancias que la que están obteniendo en la empresa bajo análisis.

Por lo tanto, el flujo de caja es un instrumento de gestión valiosa, porque permite apreciar la estructura de ingresos y egresos de efectivo y su respectiva variación en el tiempo. En las proyecciones económico financieras del negocio, el pronóstico del flujo de caja es fundamental en la toma de decisiones.

\section{INDICADORES PATRIMONIALES}

Como resultado de los indicadores de gestión operativos y económico financieros, se puede apreciar cuál es el valor del negocio para sus dueños, llamados también inversionistas o accionistas. Una apreciación estática del valor de una compañía se refleja en el valor del patrimonio neto, cuenta que forma parte del balance general de la empresa.

La empresa, con el activo que posee genera ingresos, con los cuales paga sus costos operativos, cancela sus obligaciones financieras (deuda) y cumple con sus obligaciones tributarias (participación laboral e impuesto a la renta). El nivel de endeudamiento influye en la capacidad de generar ingresos netos para sus accionistas, asimismo, permite definir la posibilidad de asumir nuevas obligaciones financieras, sea para crecer, modernizarse o diversificarse.

De esta manera, en el balance general a una fecha determinada, se tiene la composición de lo que posee una empresa (activo), las obliga- ciones que tiene (pasivo) y la porción del negocio que realmente pertenece a los dueños (patrimonio neto). Así, entre otros indicadores, se tiene el nivel de endeudamiento o palanqueo financiero, que es la relación entre el pasivo total y el patrimonio neto, llamada también relación Deuda / Capital; cuyo valor depende de una serie de factores, como el tipo de negocio, de los accionistas y de los acreedores.

La interpretación de la relación Deuda / Capital (D/C) es similar al de otros indicadores de su mendable agotar esfuerzos para su interpretación, toda vez que pueden existir razones operativas o de otra índole que sustenten las variaciones que se observen en el tiempo.

Como ejemplo mostramos en el cuadro $\mathrm{N}^{\circ} 3$, la evolución de la relación $\mathrm{D} / \mathrm{C}$ de dos empresas dedicadas al negocio de generación eléctrica, una de propiedad íntegramente del Estado y la otra de propiedad de inversionistas privados. tipo, pero que al comparar negocios, es reco-

Cuadro $\mathrm{N}^{\circ} 3$

Relación Deuda / Capital

\begin{tabular}{|l|c|c|c|}
\hline \multicolumn{1}{|c|}{ Empresa } & 1997 & 1998 & 1999 \\
\hline \hline Empresa de Generación del Estado & 1,2 & 1,3 & 0,8 \\
Empresa de Generación Privada & 0,2 & 0,3 & 0,5 \\
\hline
\end{tabular}




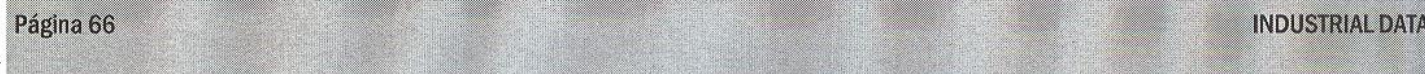

A simple vista, la empresa privada tiene menor palanqueo financiero, a pesar que éste se viene incrementando anualmente; en cambio, la empresa estatal registra una reducción importante en el último año. La interpretación de estas variaciones requiere de mayor información de de talle. Para el ejemplo mostrado, una aproximación es la siguiente:

- La empresa privada, antes del año 1997 era íntegramente de propiedad del Estado, fue transferida al sector privado mediante Licitación Pública Internacional. Para su venta, previamente se saneó toda la deuda de mediano y largo plazo, vale decir que la empresa fue transferida prácticamente sin deuda. Gracias a su mínimo nivel de endeudamiento, la empresa ha venido creciendo sostenidamente, incrementando su capacidad instalada, con sus propios recursos, aportes de capital de los nuevos dueños y con financiamientos de mediano y largo plazo. El resultado a fines del año 1999, cuenta con una potencia instalada cercana a la empresa estatal. Al cierre del referido ejercicio, el $33 \%$ del activo es financiado por acreedores y el otro $67 \%$ por los accionistas privados.

- La empresa estatal, ha venido aplicando diversas reorganizaciones mediante la escisión en diversas empresas generadoras, transmisoras y distribuidores de energía eléctrica. En todas ellas, se transfería activos, pero sin deuda. Esta situación explica en parte el alto nivel de endeudamiento hasta el año 1998. Para compensar esta situación el Ministerio de Economía y Finanzas en el año 1999 dispuso que el Estado asuma las obligaciones financieras relacionadas a los activos transferidos, por lo que el nivel de endeudamiento se reduce a 0,8 veces, con lo cual, el $44 \%$ del activo total es financiado con deuda y el $56 \%$ con capital propio. Esta empresa ha realizado importantes inversiones de reposición y modernización para mejorar su capacidad efectiva y mantiene e liderazgo en el sector. Es una buena oportunidad para el sector privado, en el caso que el Estado decidiese por su privatización.

\section{CONCLUSIONES}

Los indicadores de gestión deben analizarse en forma integrada, tanto a lo operativo, como en los aspectos económico, financiero y patrimo nial. Los estados financieros proporcionan una información elemental para el análisis, lo cua requiere complementarse con indicadores de gestión operativa, relacionadas a compras, producción, ventas y administración.
Los éxitos de la gestión de un negocio no sólo dependen del esfuerzo interno de todos los integrantes de la organización, sino también del entorno o medio ambiente empresarial; lo que debe tenerse presente cuando se comparan los resultados entre las empresas, para que las apreciaciones sean objetivas y ajustadas a la realidad. 